International Journal of Pure and Applied Mathematics

Volume 89 No. 1 2013, 89-94

ISSN: 1311-8080 (printed version); ISSN: 1314-3395 (on-line version)

url: http://www.ijpam.eu

doi: http://dx.doi.org/10.12732/ijpam.v89i1.10

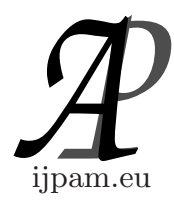

\title{
END BEHAVIOR ANALYSIS FOR SOLUTIONS OF LIMIT AT INFINITY
}

\author{
Annop Kaewkhao ${ }^{1}$, Somkid Intep ${ }^{2 \S}$ \\ ${ }^{1,2}$ Department of Mathematics \\ Faculty of Science \\ Burapha University \\ Chonburi, 20131, THAILAND
}

\begin{abstract}
In this paper, we study properties of function leaders and establish useful theorems to determine limits of functions that have indeterminate form, $\frac{\infty}{\infty}$ and $\infty-\infty$, as $x$ tend to infinity.
\end{abstract}

AMS Subject Classification: 26A06, 26A03

Key Words: end behavior, function leader, indeterminate form

\section{Introduction}

A limit determination of a function is not only one of the important things in Calculus, but also essential tool in applied mathematics. Many times we have to confront whether a function, say $f(x)$, converges to a real constant, $L$, when $x$ is extremely large. If it does, we can write that $\lim _{x \rightarrow \infty} f(x)=L$, and call that $f$ has the limit $L$ as $x$ tend to infinity. However, if $f(x)$ and $g(x)$ are increased without bound when $x$ is very large, no one can guarantee that $\lim _{x \rightarrow \infty} \frac{f(x)}{g(x)}$ will exist or not. Precisely, we call the limit in such this case that an indeterminate form.

Received: August 1, 2013

(c) 2013 Academic Publications, Ltd.

$\S$ Correspondence author url: www.acadpubl.eu 
Apart from L'Hospital's rule, there is no formal prove in investigating the end behavior of $f$ and $g$ to determine $\lim _{x \rightarrow \infty} \frac{f(x)}{g(x)}=\frac{\infty}{\infty}$, even in the good reference books in calculus such [1] and [2].

In this article, we will explain what is meant by the word "end behavior", and state useful theorems for finding the limit in the form $\lim _{x \rightarrow \infty} \frac{f(x)}{g(x)}=\frac{\infty}{\infty}$ in the next section.

\section{End Behavior Analysis}

If a function $g(x)$ has the same behavior as $f(x)$ when $x$ tend to infinity, we will call that $f$ and $g$ has the same end behavior. To make analysis compact, we set up a definition of a function leader, which has the same end behavior as the function. Then, we study useful properties of leader, and give some example to convince.

Definition 1. Let $f: \mathbb{R} \rightarrow \mathbb{R}$ and $a, r \in \mathbb{R}$. We call $a x^{r}$, denote by $\lceil f(x)\rceil$, to be a leader of $f$ if $\lim _{x \rightarrow \infty} \frac{f(x)}{a x^{r}}=1$, i.e., $\lim _{x \rightarrow \infty} \frac{f(x)}{\lceil f(x)\rceil}=1$. We also call $r$ to be a degree of $\lceil f(x)\rceil$ which denoted by $\circ\lceil f(x)\rceil$.

By the definition above, it is easy to see that $\lim _{x \rightarrow \infty} \frac{\lceil f(x)\rceil}{f(x)}=1$.

Remark 2. Any real-valued function is not necessary to have a leader, however, if one has, there is unique.

Example 3. Let $f(x)=\sin \frac{1}{x}$. Since

$$
\lim _{x \rightarrow \infty} \frac{\sin \frac{1}{x}}{\frac{1}{x}}=1
$$

the leader of $f(x)$ is $x^{-1}$.

Let $g(x)=e^{x}$. There are no real constants, $a$ and $r$, such that

$$
\lim _{x \rightarrow \infty} \frac{e^{x}}{a x^{r}}=1
$$

Hence, $g(x)$ does not have a function leader.

Since a function could have a unique leader, we find following nice properties: 
Proposition 4. Let $f$ and $g$ be real-valued functions which have leaders, then
a) $\left\lceil\frac{1}{f}\right\rceil=\frac{1}{\lceil f\rceil}$
b) $\lceil f \cdot g\rceil=\lceil f\rceil \cdot\lceil g\rceil$, and
c) $\left\lceil\frac{f}{g}\right\rceil=\frac{\lceil f\rceil}{\lceil g\rceil}$.

Theorem 5. Let $f$ and $g$ be real-valued functions which have leaders such that $\lceil f\rceil+\lceil g\rceil \neq 0$. Then

$$
\lceil f+g\rceil=\left\{\begin{array}{cl}
\lceil f\rceil & \text { when } \circ\lceil f\rceil>\circ\lceil g\rceil, \\
\lceil g\rceil & \text { when } \circ\lceil f\rceil<\circ\lceil g\rceil, \\
\lceil f\rceil+\lceil g\rceil & \text { when } \circ\lceil f\rceil=\circ\lceil g\rceil .
\end{array}\right.
$$

Proof. Suppose $\lceil f\rceil=a x^{r}$ and $\lceil g\rceil=b x^{s}$ such that $a x^{r}+b x^{s} \neq 0$. Obviously, the leader of $f+g$ is $\lceil f\rceil$ or $\lceil g\rceil$ when $\circ\lceil f\rceil \neq \circ\lceil g\rceil$. Now, consider the case $\circ\lceil f\rceil=\circ\lceil g\rceil$, i.e., $r=s$. Since $\lceil f\rceil+\lceil g\rceil \neq 0$, it follows that $a+b \neq 0$. So, we have

$$
\frac{f+g}{\lceil f\rceil+\lceil g\rceil}=\frac{f+g}{(a+b) x^{r}}=\frac{f}{(a+b) x^{r}}+\frac{g}{(a+b) x^{r}} .
$$

Hence,

$$
\lim _{x \rightarrow \infty} \frac{f+g}{\lceil f\rceil+\lceil g\rceil}=\frac{a}{a+b}+\frac{b}{a+b}=1,
$$

and therefore $\lceil f+g\rceil=\lceil f\rceil+\lceil g\rceil$.

Lemma 6. Let $\lim _{x \rightarrow \infty} f(x)=L>0$. Then

$$
\lim _{x \rightarrow \infty} g(x)=\infty \text { if and only if } \lim _{x \rightarrow \infty} f(x) g(x)=\infty .
$$

Proof. Suppose $\lim _{x \rightarrow \infty} g(x)=\infty$. Let $N \in \mathbb{N}$, by the hypotheses, there are $N_{0}$ and $N_{1} \in \mathbb{N}$ such that

$$
\begin{aligned}
& |f(x)-L|<\frac{L}{2} \quad \text { for all } x \geq N_{0}, \\
\text { i.e., } \quad & \frac{L}{2}<f(x)<\frac{3 L}{2},
\end{aligned}
$$


and $\quad g(x)>\frac{2 N}{L}$ for all $x \geq N_{1}$.

Now, let $N_{\varepsilon}=\max \left\{N_{0}, N_{1}\right\}$. We have that

$$
f(x) g(x)>\frac{L}{2} \cdot \frac{2 N}{L}=N \text { for all } x \geq N_{\varepsilon} .
$$

Therefore, $\quad \lim _{x \rightarrow \infty} f(x) g(x)=\infty$. that

For conversion, suppose $\lim _{x \rightarrow \infty} f(x) g(x)=\infty$ so that there is $N_{3} \in \mathbb{N}$ such

$$
f(x) g(x)>\frac{3 L N}{2} \text { for all } x \geq N_{3} .
$$

Let $N_{4}=\max \left\{N_{0}, N_{3}\right\}$. Then, by (1), $g(x)>N$ for all $x \geq N_{4}$.

It follows that $\lim _{x \rightarrow \infty} g(x)=\infty$.

Theorem 7. Let $f$ be a real-valued function which has leader. Then

$$
\lim _{x \rightarrow \infty} f(x)=\lim _{x \rightarrow \infty}\lceil f(x)\rceil .
$$

Proof. Consider,

$$
\lim _{x \rightarrow \infty} f=\lim _{x \rightarrow \infty} \frac{f}{\lceil f\rceil} \cdot\lceil f\rceil .
$$

If $\lim _{x \rightarrow \infty}\lceil f\rceil$ exists,

$$
\lim _{x \rightarrow \infty} f=\lim _{x \rightarrow \infty}\lceil f\rceil .
$$

On the other hand, if $\lim _{x \rightarrow \infty}\lceil f\rceil=\infty$, then, by Lemma 6 ,

$$
\lim _{x \rightarrow \infty} f=\lim _{x \rightarrow \infty}\lceil f\rceil .
$$

Therefore, the proof completed.

Now we are ready to state the useful theorem to find the limit of the form $\lim _{x \rightarrow \infty} \frac{f(x)}{g(x)}=\frac{\infty}{\infty}$.

Theorem 8. Let $f$ and $g$ be real-valued functions which have leaders. If $\lim _{x \rightarrow \infty} f(x)=\infty$ and $\lim _{x \rightarrow \infty} g(x)=\infty$, then

$$
\lim _{x \rightarrow \infty} \frac{f(x)}{g(x)}=\lim _{x \rightarrow \infty} \frac{\lceil f(x)\rceil}{\lceil g(x)\rceil} .
$$


Proof. It is straightforward from Theorem 7 and Proposition 4 that

$$
\lim _{x \rightarrow \infty} \frac{f}{g}=\lim _{x \rightarrow \infty}\left\lceil\frac{f}{g}\right\rceil=\lim _{x \rightarrow \infty} \frac{\lceil f\rceil}{\lceil g\rceil}
$$

Example 9. It is easy to check that $2 x^{3}$ is the leader of

$$
f(x)=\sqrt{4 x^{6}+\sqrt{5 x^{7}+\sqrt{6 x^{8}}}}
$$

and $x^{3}$ is the leader of $g(x)=\left(x^{3 / 2}+2 x+1\right)^{2}$. Therefore, by Theorem 8 ,

$$
\lim _{x \rightarrow \infty} \frac{f(x)}{g(x)}=\lim _{x \rightarrow \infty} \frac{2 x^{3}}{x^{3}}=2 .
$$

From this example, we can see that in finding the solution our method is more convenient than the L'Hospital's rule as the quotient, $\frac{f^{\prime}(x)}{g^{\prime}(x)}$, is complicated.

Remark 10. If $\lim _{x \rightarrow \infty}(f(x)-g(x))=\infty-\infty$, then we multiply both the numerator and the denominator by its conjugation, $f(x)+g(x)$. The limit, then, transforms to $\frac{\infty}{\infty}$. We can now apply Theorem 8 to get a limit solution. For example,

$$
\begin{aligned}
& \lim _{\mathrm{x} \rightarrow \infty} \sqrt{x^{4}+\sqrt{x^{6}+x}}-\left(x^{2}-x\right) \\
& =\lim _{\mathrm{x} \rightarrow \infty}\left[\sqrt{x^{4}+\sqrt{x^{6}+x}}-\left(x^{2}-x\right)\right] \cdot \frac{\left[\sqrt{x^{4}+\sqrt{x^{6}+x}}+\left(x^{2}-x\right)\right]}{\sqrt{x^{4}+\sqrt{x^{6}+x}}+\left(x^{2}-x\right)} \\
& =\lim _{\mathrm{x} \rightarrow \infty} \frac{\sqrt{x^{6}+x}+2 x^{3}-x^{2}}{\sqrt{x^{4}+\sqrt{x^{6}+x}}+\left(x^{2}-x\right)} \\
& =\lim _{\mathrm{x} \rightarrow \infty} \frac{\sqrt{x^{6}}+2 x^{3}}{\sqrt{x^{4}}+x^{2}}=\lim _{\mathrm{x} \rightarrow \infty} \frac{3 x}{2}=\infty .
\end{aligned}
$$




\section{Conclusion}

We established the useful theorem to find limits as $x$ tend to infinity for any functions that have their leaders, especially a function which has indeterminate form $\frac{\infty}{\infty}$ or $\infty-\infty$. We also emphasize that our method is easier and more convenient than other methods.

Interestingly, what are necessary and sufficient conditions to ensure that a function can has a leader?

\section{Acknowledgements}

The authors would like to thank the Faculty of science, Burapha university, Thailand for financial support.

\section{References}

[1] H. Anton, I. Bivens, S. Davis, Calculus, 8-th Edition, John Wiles \& Sons, USA (2005).

[2] G.B. Tomas, R.L. Finney, Calculus and Analytic Geometry, 8-th Edition, Addison-Wesley, USA (1993). 